\title{
Modelling the singular mineralization process due to fluid pressure fluctuations
}

Yihui Xiong ${ }^{1}$, Renguang Zuo ${ }^{1 *}$

${ }^{1}$ State Key Laboratory of Geological Processes and Mineral Resources, China University of Geosciences, Wuhan 430074, China (*correspondence: zrguang@cug.edu.cn)

Singular physical and chemical processes may lead to anomalous amounts of energy release or mass accumulation within a narrow spatial-temporal interval. Mineralization in the Earth's crust can be regarded as a type of singular process as a result of large amounts of mass accumulation with element enrichment characterized by fractal or multifractal. Fluid pressure fluctuations induced by earthquake or fault ruptures are frequently responsible for metal solubility, and trigger transient physical and chemical variations in oreforming fluids that can enhance the mineralization process through phase separation. In this model, the mineral precipitation process during rapid fluid pressure fluctuation due to fault failure is incorporated into a cellular automaton model to explore the basic nonlinear behaviors within the singular mineralization processes. A multifractal model and geostatistics are used to quantify the resulting spatial structures and their variation of geochemical patterns that are the end products of the mineralization process. The distribution of metal concentrations evolves from local depletion to local enrichment patterns among the whole system according to multifractal spectrum. A similar phenomenon emerged in the evolution of spatial autocorrelation in the geochemical pattern which decreased first and then increased. This transition can be attributed to the coalescence of sub-networks with different fluid pressures that result in a wider range of fluid pressure fluctuations and mineral precipitation in the form of clusters. These suggest that cycles in the evolution of the fluid pressure system governed by local permeability are able to reproduce the repeated mineralization superposition process, and generate a complex geochemical pattern characterized by a multifractal model. The nonlinear behavior shown in this model exhibits scale-invariance and has self-organized critical thresholds where mineral phase transitions are induced by fault failure. All of these results suggest that the simplified model can give a near-perfect expression of the singular mineralization process, and can generate complex multifractal geochemical structures. 